Classification

Physics Abstracts

05.30

\title{
ÉTUDE GÉNÉRALE DES SONDES DE MESURE INDUCTIVE DE COURANTS. II. RÉPONSE AUX HAUTES FRÉQUENCES. COMPARAISON DES DIVERS TYPES DE SONDES
}

\author{
F. RIOUX-DAMIDAU et C. RIOUX \\ Laboratoire de Physique des Plasmas, bâtiment 214, 91, Orsay
}

(Reçu le 28 juin 1972)

\begin{abstract}
Résumé. - On étudie tout d'abord la réponse d'une sonde lorsque l'induction magnétique créée par le courant qu'elle mesure n'est pas axi-symétrique et on en déduit la valeur de la coupure haute fréquence. Puis on compare les divers types de sondes en associant ces résultats avec ceux obtenus en (1).
\end{abstract}

Abstract. - The probe response is studied in the case when the magnetic induction produced by the measured current is non axisymmetric and the high frequency probe cut-off is deduced. Different types of probes are then compared by associating these results with those derived in (1).

I. Introduction. - Dans un précédent article (1) que nous désignerons désormais par RP 1, nous avions commencé une étude générale des sondes inductives de mesure de courant. Ces sondes comportaient $N$ spires d'un fil métallique bobiné sur un tore, qui entourait le conducteur $\mathrm{C}$ dans lequel circule le courant $J$ à mesurer. Leur fonctionnement dépendant beaucoup de leur structure, nous avions été amenés à distinguer trois types de sondes :

- Les ceintures de Rogowski (CR), aux bornes desquelles on mesure directement la tension engendrée par la variation de J. Le signal obtenu est proportionnel à $\mathrm{d} J / \mathrm{d} t$.

- Les sondes à écran total (SET) et les transformateurs d'intensité (TI) qui ont une résistance interne $\mathcal{R}$ très faible et qui sont parcourus par un courant $i=\mathfrak{J} / N$.

- Les sondes à débit interne (SDI) dont la résistance interne est assez élevée et qui sont parcourues par un courant $i$ assez fort. Elles permettent de mesurer $\mathfrak{J}$ et $\mathrm{d} \mathfrak{J} / \mathrm{d} t$.

Nous avions cherché le domaine d'utilisation possible de ces sondes lorsque le courant suivait l'axe du tore, c'est-à-dire lorsque l'induction magnétique $\mathfrak{B}_{1}$ créée par ce courant possédait une complète symétrie de révolution.

En fait, l'induction "magnétique est rarement axisymétrique et toutes les spires de la sonde ne sont pas soumises au même flux. Les phénomènes de propagation deviennent alors importants dans la détermination de la réponse de la sonde aux hautes fréquences. Nous allons les étudier dans ce deuxième article.

Remarquons cependant que, dans une ceinture de Rogowski, les points de sortie A et B (cf. Fig. 1 de RP 1) de la mesure sont portés à des potentiels diffé-

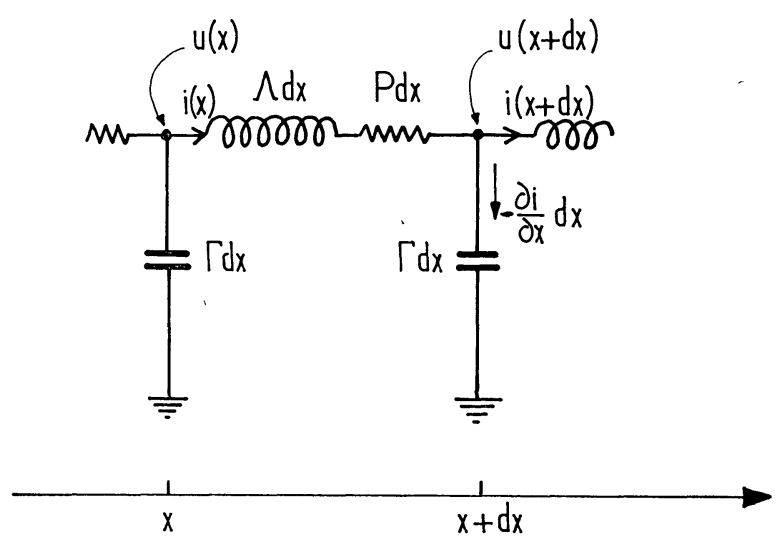

FIG. 1.

rents, ce qui engendre une dissymétrie angulaire importante; des ondes stationnaires prennent naissance dans la sonde même lorsque le champ $\mathfrak{B}_{1}$ est de révolution. Un calcul simplifié nous a permis dans RP 1 d'évaluer la fréquence de ces ondes en fonction de la capacité $C_{0}$ de l'appareil de mesure placé entre A et $B$. Nous ne considérons pas la limitation apportée par l'anisotropie du champ car on peut montrer que le domaine d'utilisation n'est pas réduit par rapport au précédent. L'étude des ondes stationnaires ne portera donc que sur les sondes parcourues par un courant $i$ non nul (TI, SET, SDI) pour lesquelles la mesure est alors un quasi-courtcircuit qui ne perturbe que très faiblement la symétrie de révolution de la structure.

En complétant alors avec les résultats obtenus dans RP 1, nous pourrons préciser le domaine d'utilisation et la meilleure structure de chaque type de sonde puis comparer leurs performances respectives. 
II. Influence de l'anisotropie du champ extérieur. 1. EXAMEN DU PROBLÈme POSÉ. - Pour simplifier l'exposé, considérons une sonde dont la structure possède une parfaite symétrie de révolution. Cet objet se présente sous la forme d'un solénoïde torique pour lequel on peut définir une inductance $\Lambda$, une capacité $\Gamma$ et une résistance $P$ par unité de longueur. Si $r$ et $R$ sont respectivement les petit et grand rayons du tore, $s$ et $\rho$ la section et la résistivité du fil formant les $N$ spires, on montre que :

$$
\begin{aligned}
& \Lambda \simeq \mu_{0}\left(\frac{N}{2 \pi R}\right)^{2} \pi r^{2} \\
& P \simeq \rho \frac{r N}{s R} .
\end{aligned}
$$

La valeur de $\Gamma$ dépend de la structure précise de la sonde. Dans le cas où cette dernière comporte un fil de retour $\mathrm{A}^{\prime} \mathrm{A}$ (cf. Fig. $1 \mathrm{RP}$ 1) de rayon $r_{\mathrm{f}}$, la capacité est principalement celle qui existe entre le bobinage et ce fil. L'on a alors :

$$
\Gamma \simeq \frac{2 \pi \varepsilon_{0}}{\log \left(r / r_{\mathrm{f}}\right)}
$$

S'il n'y a pas de fil de retour, nous considérerons la capacité par rapport à une masse lointaine et nous écrirons :

$$
\Gamma \simeq \frac{2 \pi \varepsilon_{0}}{\log (R / r)}
$$

Ces deux formules seront valables pour les SDI et les SET. En ce qui concerne les TI, ces derniers comportent un noyau magnétique de perméabilité $\mu=\mu_{0} \mu_{\mathrm{r}}$ séparé du bobinage par un isolant de permittivité électrique $\varepsilon=\varepsilon_{0} \varepsilon_{\mathrm{r}}$. Le rayon du noyau étant $r_{0}$ et le rayon interne du bobinage $r_{0}$ (Fig. 3 RP 1), la capacité par unité de longueur entre le noyau et le bobinage vaut :

$$
\Gamma \simeq \frac{2 \pi \varepsilon}{\log \left(r_{0} / r_{0}^{\prime}\right)}
$$

et l'inductance par unité de longueur

$$
\Lambda \simeq \mu\left(\frac{N}{2 \pi R}\right)^{2} \pi r^{2} .
$$

La sonde est placée dans l'induction $B_{1}(x, t)$ créée par le courant $\mathfrak{J}, x=R \theta$ représentant l'abscisse mesurée le long du tore. La donnée de $B_{1}$ suffit pour calculer la réponse de la sonde; nous utiliserons plus précisément la quantité qui lui est directement reliée :

$$
S(x, t)=\frac{2 \pi R B_{1}(x, t)}{\mu_{0} N}
$$

$\mathcal{S}(x, t)$ est le terme source qui détermine le courant $i=i(x, t)$ et la tension $u=u(x, t)$ (cf. Fig. 1) le long de la sonde.

Lorsque l'induction magnétique et par suite le terme source sont indépendants de $x$, le courant $i(x, t)$ est lui aussi indépendant de $x$. On a $: i(x, t)=i_{0}(t)$; la mesure de $i_{0}$ donne directement $J$.
Lorsque au contraire la distribution de $B_{1}$ est anisotrope, le courant $i(x, t)$ dépend effectivement de $x$. Comme nous l'avons montré dans RP $1, \mathfrak{J}$ est directement lié à la valeur moyenne du courant qui est égale à $i_{0}(t)$, mais la mesure ne donne que $i\left(x_{\mathrm{m}}, t\right)$ où $x_{\mathrm{m}}$ repère la position de l'impédance de mesure. La relation entre $i\left(x_{\mathrm{m}}, t\right)$ et $i_{0}(t)$ est $a$ priori inconnue. L'erreur que l'on commet sur la détermination de $i_{0}(t)$ est donc :

$$
\frac{\Delta i}{i_{0}}=\frac{i\left(x_{\mathrm{m}}, t\right)-i_{0}(t)}{i_{0}(t)} .
$$

Le problème consiste à évaluer cette erreur. On admet que l'erreur sur $\mathfrak{J}$ est du même ordre de grandeur.

2. Calcul de l'erreur sur $i$. - Les calculs d'évolution de $i(x, t)$ et de $u(x, t)$ s'établissent facilement en se reportant à la figure 1 . On obtient :

$$
\begin{aligned}
& -\frac{\partial u}{\partial x}=\Lambda\left(\frac{\partial i}{\partial t}+\frac{\partial \mathcal{S}}{\partial t}\right)+P i \\
& -\frac{\partial i}{\partial x}=\Gamma \frac{\partial u}{\partial t}
\end{aligned}
$$

soit, en éliminant $u$ entre ces deux équations.

$$
\Lambda \Gamma \frac{\partial^{2} i}{\partial t^{2}}-\frac{\partial^{2} i}{\partial x^{2}}+\Gamma P \frac{\partial i}{\partial t}=-\Lambda \Gamma \frac{\partial^{2} \delta}{\partial t^{2}}
$$

Le problème consiste à calculer $i$ lorsque $\delta$ est donné. Pour cela, nous décomposerons $\delta$ et $i$ en série de Fourier par rapport à $x$ et $t$ et nous écrirons :

$$
\begin{gathered}
\mathcal{S}=\text { Réel } \sum_{\omega, p} S_{\omega_{p}}=\operatorname{Réel~} \sum_{\omega, p} s_{\omega_{p}} \exp \left[j\left(\omega t+p \frac{x}{R}\right)\right] \\
i=\operatorname{Réel~} \sum_{\omega, p} i_{\omega_{p}}=\operatorname{Réel~} \sum_{\omega, p} \mathrm{i}_{\omega_{p}} \exp \left[j\left(\omega t+p \frac{x}{R}\right)\right]
\end{gathered}
$$

$p$ est un nombre entier pouvant varier de 0 à l'infini ; $R / p$ représente la longueur d'onde pour le $p$-ième harmonique ; $\sum_{\omega}$ peut éventuellement représenter une intégration et non une sommation discrète.

En absence d'anisotropie, la décomposition de $S$ ne comprend que les termes $p=0$; le courant $i$ est indépendant de $x$ et égal à $i_{0}(t)$, soit :

$$
i_{0}(t)=\operatorname{Réel~} \sum_{\omega} \mathrm{i}_{\omega_{0}} \mathrm{e}^{j \omega t} \text {. }
$$

Les anisotropies, lorsqu'elles existent, sont décrites par les termes $s_{\omega_{p}}$ et $\mathrm{i}_{\omega_{p}}$ et l'erreur sur la mesure est donnée par:

$$
\begin{aligned}
\frac{\Delta i}{i_{0}} & =\frac{i\left(x_{\mathrm{m}}, t\right)-i_{0}(t)}{i_{0}(t)} \\
& =\operatorname{Réel} \frac{\sum_{\omega} \sum_{p=1}^{\infty} \mathrm{i}_{\omega_{p}} \exp \left[j\left(\omega t+p \frac{x_{\mathrm{m}}}{R}\right)\right]}{\sum_{\omega} \mathrm{i}_{\omega_{0}} \mathrm{e}^{j \omega t}} .
\end{aligned}
$$


Lorsqu'une des pulsations $\omega$. prédomine dans le signal, cette erreur est telle que :

$$
\frac{\Delta i}{i_{0}} \simeq \operatorname{Réel} \frac{\sum_{p=1}^{\infty} \mathrm{i}_{\omega_{p}} \exp \left(j p \frac{x_{\mathrm{m}}}{R}\right)}{\mathrm{i}_{\omega_{0}}} \leqslant \sum_{p=1}^{\infty}\left|\frac{\mathrm{i}_{\omega_{p}}}{\dot{\mathrm{i}}_{\omega_{0}}}\right| .
$$

Pour connaître une majoration de l'erreur, nous sommes ainsi amenés à calculer $\left|i_{\omega_{p}} / i_{\omega_{0}}\right|$, ce que nous ferons en déterminant le signal de réponse $i_{\omega_{p}}$ correspondant à une source $s_{\omega_{p}}$. Pour cela, nous écrivons l'éq. (6) pour le terme $(\omega, p)$ de la décomposition de Fourier ce qui donne :

$$
\left(-\Lambda \Gamma \omega^{2}+\frac{p^{2}}{R^{2}}+j \Gamma P \omega\right) \mathrm{i}_{\omega_{p}}=\Lambda \Gamma \omega^{2} s_{\omega_{p}} .
$$

Nous introduisons les pulsations caractéristiques :

$$
\omega_{c}=\frac{P}{\Lambda}=\frac{\Re}{L}
$$

et

$$
\omega_{1}=\frac{1}{R \sqrt{\Lambda \Gamma}}=\frac{2 \pi}{\sqrt{L C}}, \quad \omega_{p}=p \omega_{1}
$$

où $\mathcal{R}, L$ et $C$ sont la résistance de la sonde, sa self et sa capacité par rapport à une masse lointaine. $1 / \omega_{c}$ est le temps caractéristique de la pénétration du champ magnétique dans la sonde tandis que $\omega_{1}$ est la pulsation correspondant au premier harmonique des phénomènes de résonance dans la sonde et $\omega_{p}$ celle correspondant au $p$-ième harmonique.

Explicitons les expressions (8). Nous avons, à partir de (1), (2) et (1a) :

$$
\omega_{c}=\frac{R}{L}=\frac{4 \pi}{\mu_{0} \mu_{r}} \cdot \frac{\rho}{s N} \cdot \frac{R}{r}
$$

où $\mu_{r}=1$ pour les SDI et SET et $\mu_{r} \neq 1$ pour les TI.

L'expression de $\omega_{1}$ dépend de la structure précise de la sonde par l'intermédiaire de $\Gamma$ et $\Lambda$.

Pour nous ramener à une expression générale, nous remarquerons que nous pouvons écrire dans tous les cas :

$$
\sqrt{L C}=\frac{2 \pi N r}{\gamma}
$$

où $\gamma$ est un paramètre qui dépend de la sonde et qui a les dimensions d'une vitesse :

Pour les SDI et les SET nous avons deux expressions possibles de $\gamma$ :

$$
\gamma=c \sqrt{2 \log \frac{r}{r_{\mathrm{f}}}}
$$

lorsque la sonde comporte un fil de retour et

$$
\gamma=c \sqrt{2 \log \frac{R}{r}}
$$

lorsque la sonde ne comporte pas de fil de retour.
Pour les TI $\gamma$ est donné par :

$$
\gamma=\frac{1}{\sqrt{\varepsilon \mu}} \sqrt{2 \log \frac{r_{0}}{r_{0}^{\prime}}} .
$$

Nous pouvons donc finalement écrire :

$$
\omega_{1}=\frac{\gamma}{N r} .
$$

Nous voyons en particulier que $2 \pi / \omega_{1}$ est le temps mis par un signal pour parcourir toute la longueur du fil de la sonde à la vitesse $\gamma$. Pour une SDI ou une SET, cette vitesse est en général comprise entre 2 et $4 c$; nous prendrons $\gamma=3,3 c=10^{9} \mathrm{~m} / \mathrm{s}$. Pour un TI, nous admettrons que $r_{0} / r_{0}^{\prime} \simeq 1,1, \varepsilon \simeq 4$ et $\mu_{r} \simeq 10^{4}$, ce qui conduit à $\gamma \simeq 6 \times 10^{5} \mathrm{~m} / \mathrm{s}$.

Avec ces nouvelles notations, l'éq. (9) prend la forme simplifiée

$$
\left(-1+\frac{\omega_{p}^{2}}{\omega^{2}}+j \frac{\omega_{c}}{\omega}\right) \mathrm{i}_{\omega_{p}}=s_{\omega_{p}} .
$$

On en déduit :

$$
\frac{\mathrm{i}_{\omega_{p}}}{\mathrm{i}_{\omega_{0}}}=\frac{1-j\left(\omega_{c} / \omega\right)}{1-\omega_{p}^{2} / \omega^{2}-j\left(\omega_{c} / \omega\right)} \cdot \frac{s_{\omega_{p}}}{s_{\omega_{0}}}
$$

et

$$
\left|\frac{i_{\omega_{p}}}{i_{\omega_{0}}}\right|=\eta\left|\frac{s_{\omega_{p}}}{s_{\omega_{0}}}\right|
$$

avec

$$
\eta=\sqrt{\frac{1+\left(\omega / \omega_{c}\right)^{2}}{1+\left(\omega / \omega_{c}\right)^{2}\left(1-\omega_{p}^{2} / \omega^{2}\right)^{2}}} .
$$

L'étude de $\eta$ va nous permettre de préciser l'influence des anisotropies.

3. ETUDE DE $\eta .-\eta\left(\omega, \omega_{p}, \omega_{c}\right)$ est une fonction homogène des 3 variables $\omega, \omega_{p}$ et $\omega_{c}$.

Pour l'étudier, nous introduirons les deux nouvelles variables

$$
\xi_{p}=\frac{\omega_{p}}{\omega_{c}}=p \cdot \frac{\mu_{0} s}{4 \pi \rho} \cdot \frac{c \sqrt{2 \log R / r}}{R}=p \frac{\mu_{0} s}{4 \pi \rho} \frac{\gamma}{R}
$$

$$
\chi=\frac{\omega}{\omega_{c}}=\frac{\omega}{L / \mathcal{R}}=\frac{\mu_{0} s}{4 \pi \rho} \cdot \frac{N r}{R} \cdot \omega
$$

$\xi_{p}$ est un paramètre caractéristique de la structure de la sonde. $\chi$ représente le rapport entre la fréquence du courant à mesurer et la constante de temps de pénétration de l'induction magnétique dans la sonde. Lorsque $\chi \leqslant 1$, nous avons affaire à une SDI; pour $\chi \geqslant 1$, nous avons une SET ou un TI. Nous utiliserons aussi le rapport : $\chi / \xi_{p}=\omega / \omega_{p}$ qui compare la fréquence du courant $\mathfrak{J}$ et les fréquences excitant les résonances spatiales de la sonde. 
L'expression de $\eta$ devient ainsi :

$$
\eta=\sqrt{\frac{1+\chi^{2}}{1+\left(\chi-\xi_{p}^{2} / \chi\right)^{2}}} .
$$

Les figures 2 et 3 représentent en coordonnées logarithmiques $\eta(\chi)$ et $\eta\left(\chi / \xi_{p}\right)$ pour diverses valeurs de $\xi_{p}$. Sur le diagramme $\eta\left(\chi / \xi_{p}\right)$ on remarque que les courbes correspondant à $\chi>1$, donc aux SET et TI, se regroupent pratiquement en une seule courbe tandis que, si $\xi_{p}>1$, elles présentent une résonance très marquée pour $\chi / \xi_{p}=1$ ( $\eta$ devenant égal à $\xi_{p}$ au maximum). Les SET et TI sont assez sensibles aux anisotropies de champ magnétique et les exaspèrent lorsque $\omega=\omega_{p}$.

Pour les SDI, nous voyons sur la figure 2 que $n \lesssim 1$. Il n'y a jamais résonance sur une anisotropie pour ces sondes.

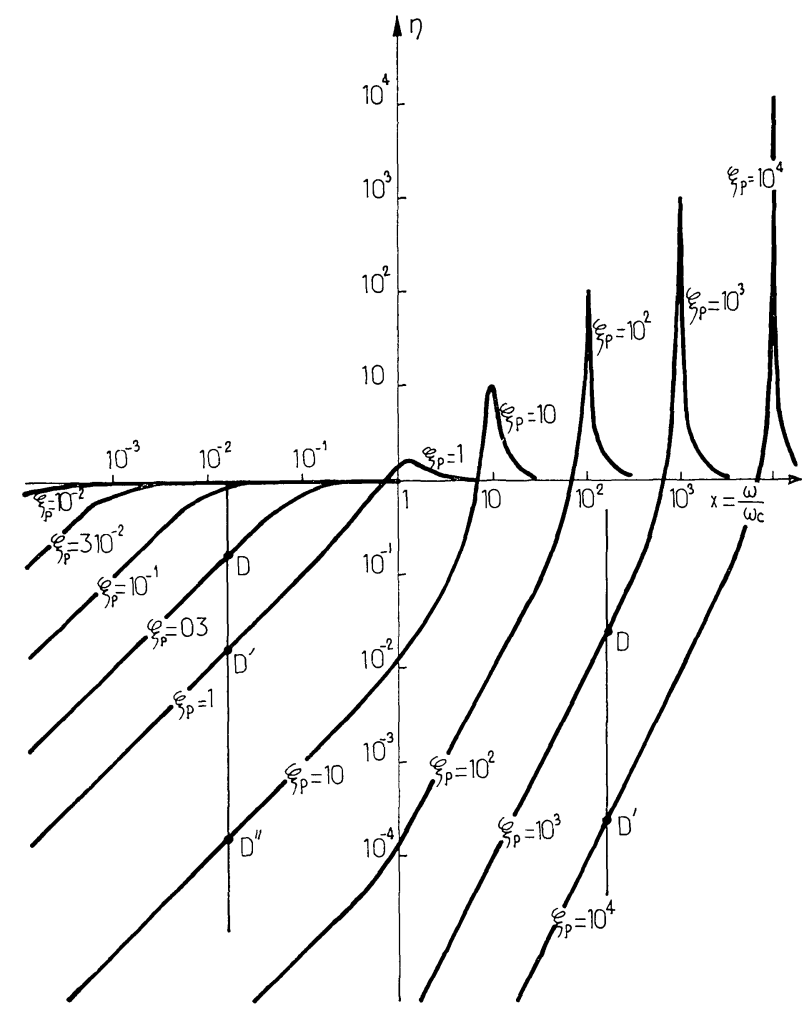

FIG. 2.

Cherchons maintenant quelles sont les conditions à observer pour que la précision d'une mesure soit au moins égale à $g$ (pour les applications numériques nous prendrons $g=10^{-2}$ comme dans RP 1). Si on admet que les anisotropies sont au plus de l'ordre de grandeur du champ magnétique, il faut que l'on vérifie constamment $\eta \leqslant g \ll 1$. Notons que pour les transformateurs d'intensité, la présence du noyau magnétique homogénéise beaucoup la distribution de $B_{1}$ tant que $B_{1}<B_{s}$, ce que nous supposons toujours réalisé.

L'anisotropie observée sur le champ magnétique est donc plus petite que celle indiquée par la valeur de $\eta$; nous imposerons dans ce cas $\eta \leqslant g(\mathrm{TI})$ avec $g(\mathrm{TI})=0,1$.

Pour une sonde et une fréquence $\omega$ données, les valeurs de $\eta$ correspondant aux différents harmoniques spatiaux déterminés sur la figure 2 en prenant l'intersection entre la verticale $\chi=\omega / \omega_{c}$ et les courbes $\eta(\chi)$ correspondant aux diverses valeurs possibles de $\xi_{p}$ pour cette sonde (points tels que $\mathrm{D}, \mathrm{D}^{\prime}, \ldots$... Nous voyons sur cette figure que si $\eta<1$ pour un harmonique, il devient de plus en plus petit lorsque le numéro $p$ de l'harmonique et par suite $\xi_{p}$ augmente. Donc si $\eta \leqslant g$ pour l'harmonique $p=1$, cette inégalité sera toujours très largement vérifiée pour les autres et nous pourrons ne considérer désormais que le premier harmonique spatial $p=1$.

Pour savoir si une mesure est valable, nous regarderons donc si le point $\chi / \xi_{1}, \eta\left(\chi / \xi_{1}\right)$ du diagramme de la figure 3 est bien situé dans la zone limitée par

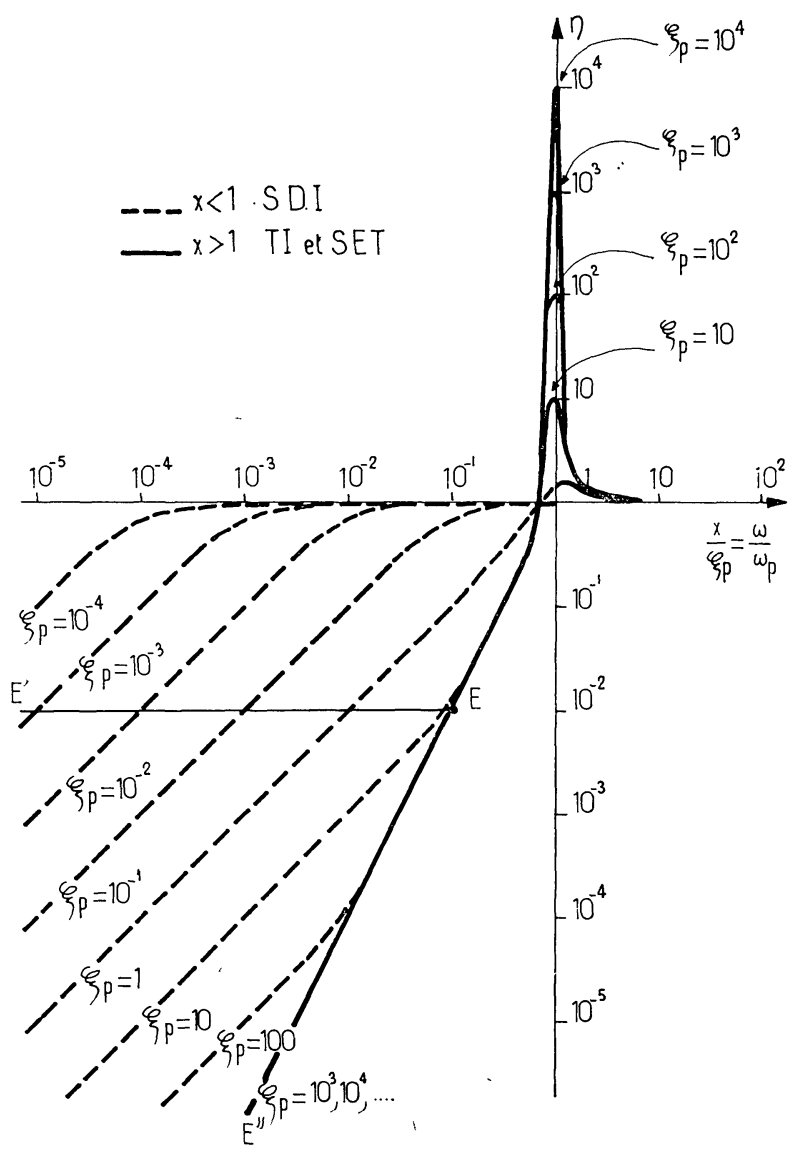

FIG. 3.

la droite $\mathrm{EE}^{\prime}(\eta=g)$ et la courbe $\mathrm{EE}^{\prime \prime}(\eta$ pour $\chi>1)$, les coordonnées du point $\mathrm{E}$ étant $\eta_{E}=g, \chi_{E} / \xi_{1}=\sqrt{g}$ : $\left(\chi_{E}=1, \xi_{1}=1 / \sqrt{g}\right.$ pour une SDI $; \chi_{E} \geqslant 1, \xi_{1} \geqslant 1 / \sqrt{g}$ pour un TI ou une SET).

Comparons alors les possibilités de diverses sondes ayant le même $\omega_{1}$, c'est-à-dire ayant sensiblement la même longueur $2 \pi r N$ de fil $\left(\omega_{1}=\gamma / N r\right.$ où $\gamma$ dépend peu de la structure précise de la sonde, cf. (11)). La plus haute fréquence mesurable $\omega_{1}$ est représentée 
sur la figure 3 pour les SDI, comme pour les SET et les TI par le point $E$; elle est telle que :

$$
\frac{\omega_{1}}{\omega_{1}}=\frac{\chi_{E}}{\xi_{1}}=\sqrt{g}
$$

soit :

$$
\omega_{1}=\frac{\sqrt{g} \gamma}{N r}
$$

Il faut remarquer que ce point $E$ n'existe pour une SDI que si $\xi_{1}=1 / \sqrt{g}$ (et $\chi=1$ ), c'est-à-dire si la sonde est construite de telle sorte que :

$$
\omega_{c}=\sqrt{g} \omega_{1} \text {. }
$$

Ceci conduit à :

$$
\frac{\rho R}{s}=\frac{\mu_{0}}{4 \pi} \sqrt{g} \gamma
$$

Soit, avec les valeurs numériques habituelles,

$$
\frac{\rho R}{s}=10 \text {. }
$$

Pour une telle sonde, compte tenu de (18) et (19), on aura donc :

$$
\omega_{c}=\omega_{1}=\sqrt{g} \omega_{1} .
$$

Si le critère de construction indiqué n'est pas respecté, la limite haute fréquence sera plus basse que celle donnée par la relation (18). Nous supposerons désormais que toutes les SDI satisfont les relations (18) et (20).

4. LimitATIONS HAUTE FRÉQUENCE GÉNÉRALES. Nous venons d'établir que la limitation haute fréquence des sondes est donnée par la relation (18) lorsqu'elle est due à l'anisotropie du champ.

Mais de plus, comme nous l'avons montré dans RP 1, il faut que l'échauffement $\Delta T$ de la sonde reste acceptable $\left(\Delta T<\Delta T_{0}\right)$. Nous avons admis pour notre étude que la sonde était soumise à une mono-impulsion de courant simulable par un courant de pulsation $\omega$ durant une demi-période. Nous avions obtenu les inéquations suivantes :

- pour les TI et SET, lorsque l'épaisseur moyenne du bobinage $e=N s / 2 \pi R$ vérifie $e>\delta$ (où $\delta$ est l'épaisseur de peau pour la pulsation $\omega$ ) :

$$
B_{e}<\left(B_{e}\right)_{M}=\left(\frac{4 \mu_{0} \bar{\omega} c_{M} \Delta T_{0}}{\pi}\right)^{1 / 2}
$$

Nous supposerons ici que cette condition $e>\delta$ est toujours réalisée par construction;

- pour les SDI

$$
r^{2} \omega B_{e}^{2}<\frac{8}{\pi} \rho \bar{\omega} c_{M} \Delta T_{0}
$$

( $\bar{\omega}$ et $c_{M}$ sont respectivement la masse spécifique et la capacité calorifique du fil du bobinage).
Remarquons que, pour établir cette dernière expression, on suppose le courant uniformément réparti dans la section du fil $s$. Il faut donc qu'il le soit en particulier pour la fréquence limite et que l'on ait :

$$
s \lesssim \pi \delta_{1}^{2}=\pi \frac{2 \rho}{\mu_{0} \omega_{1}} .
$$

En comparant à (24), nous en déduisons :

$$
2 R \lesssim N r
$$

ce qui est, en pratique, toujours réalisé.

En conclusion, les limitations haute fréquence des sondes sont données par les relations (18), (22) et.(23).

III. Utilisation et structure constructive des sondes. - Jusqu'ici, nous avons étudié séparément les divers phénomènes qui limitent l'utilisation des sondes; nous nous proposons maintenant de regrouper les résultats acquis. Nous pourrons ainsi préciser la bande passante d'une sonde, c'est-à-dire le rapport entre les fréquences maximum $\omega_{\mathrm{M}}$ et minimum $\omega_{\mathrm{m}}$ qu'elle peut mesurer (avec une précision $g=10^{-2}$ ). Nous indiquerons aussi la structure qu'il est préférable d'adopter lorsqu'un problème précis se pose.

Nous serons alors en mesure de comparer les divers types de sondes. Or, dans l'ensemble de notre étude, deux paramètres jouent un rôle essentiel $N r \omega$ et $B_{e}=\mu_{0} I / 2 \pi \dot{R}$. Pour faciliter la comparaison que nous ferons au chapitre suivant, nous allons dès maintenant nous efforcer d'exprimer nos résultats en fonction de ces paramètres. Il est bien évident que toutes les conditions de limitation des performances ne s'expriment pas à l'aide de ces seuls paramètres, mais nous verrons qu'ils nous permettent finalement de présenter un aspect suffisamment précis du problème.

1. Ceintures de Rogowski. - Les limitations des CR ont été portées sur le diagramme en

$$
\left(r \sqrt{R} B_{e}, \frac{N r \omega}{\sqrt{R}}\right)
$$

de la figure 2 de RP 1.

Rappelons que, dès que $I$ dépasse $10^{3}$ ou $10^{4} \mathrm{~A}$ (plus précisément $r \sqrt{R} B_{e} \geqslant 8 \times 10^{-6}$ ), la bande passante de la sonde est donnée par les possibilités de l'appareil de mesure de la tension qui apparaît aux bornes de la sonde puisque :

$$
\frac{\omega_{\mathrm{M}}}{\omega_{\mathrm{m}}}=\frac{U_{\mathrm{M}}}{U_{\mathrm{m}}}
$$

Avec les valeurs numériques précédemment admises, cela donne :

$$
\frac{\omega_{\mathrm{M}}}{\omega_{\mathrm{m}}}=3 \times 10^{5} .
$$

Lorsque le courant est plus petit, la fréquence supé- 
rieure $\omega_{\mathbf{M}}$ est limitée par la capacité de la mesure et la bande passante diminue.

Etant donné qu'une $\mathrm{CR}$ mesure $\mathrm{d} J / \mathrm{d} t$ c'est-à-dire $\omega I$, il est intéressant de préciser les limitations relatives à cette grandeur. De l'éq. (7a) de RP 1 , nous déduisons :

$$
\frac{U_{\mathrm{m}}}{\pi} \leqslant(N r \omega) r B_{e} \leqslant \frac{U_{\mathrm{M}}}{\pi}
$$

soit :

$$
U_{\mathrm{m}} \leqslant \frac{\mu_{0}}{2} \frac{N r^{2}}{R} I \omega \leqslant U_{M}
$$

et :

$$
1,6 \times 10^{3} \frac{R}{N r^{2}} \leqslant \omega I \leqslant 5 \times 10^{8} \frac{R}{N r^{2}}
$$

(la limitation supérieure n'est valable que lorsque $I>10^{3}$ ou $\left.10^{4} \mathrm{~A}\right)$.

Pour mesurer des courants forts à variation rapide, il faut que $N r^{2} / R=(N r / R) . r$ soit petit, il faut donc que le serrage $N r / R$ du bobinage soit faible et que $r$ soit petit. En admettant que les limites inférieures de $N r / R$ et $r$ sont : $N r / R=10, r=1 \mathrm{~mm}$, on obtient :

$$
\omega I \leqslant 5 \times 10^{10} \text {. }
$$

Les CR ne peuvent mesurer de courants très élevés à variation très rapide.

$\mathrm{Au}$ contraire, pour mesurer un $\mathrm{d} J / \mathrm{d} t$ faible, il faut une $\mathrm{CR}$ où l'on a réalisé un bobinage serré sur un gros tore $(N r / R$ et $r$ grands). En admettant les valeurs maxima, $N r / R=10^{3}, r=10 \mathrm{~cm}$, on obtient $\omega I \geqslant 15$.

La figure 4 représente le diagramme $\left(N r \omega, B_{e}\right)$ des CR. Nous y avons porté l'éq. (25) pour diverses valeurs de $r$ et l'éq. (7b) de RP 1 :

$$
\frac{N r \omega}{\sqrt{R}} \leqslant 0,1 \sqrt{\frac{2}{\mu_{0} C_{0}}}=1,25 \times 10^{7}
$$

pour diverses valeurs de $R$.

2. TI ET SET. - L'étude de l'influence des anisotropies du champ nous a montré que la fréquence supérieure d'utilisation de ces sondes est $\omega_{M}=\omega_{1}$, déterminée par la relation (18) :

$$
N r \omega_{\mathrm{M}}=\sqrt{g} \gamma
$$

où $\sqrt{g} \gamma=10^{8}$ pour les SET et $5 \times 10^{4}$ pour les TI.

La fréquence minimum est imposée par la nonpénétration du champ à l'intérieur de la sonde. D'après la figure 5 de RP 1 , on a :

$$
\frac{e r}{\delta_{\mathrm{m}}^{2}}=k,
$$

où : $\delta_{\mathrm{m}}$ est l'épaisseur de peau pour la pulsation $\omega_{\mathrm{m}}$ :

$$
\delta_{\mathrm{m}}^{2}=\frac{2 \rho}{\mu_{0} \omega_{\mathrm{m}}},
$$

$k$ est une constante de l'ordre de 50 pour les SET, $k$ dépend de la valeur de $B_{e}$ pour les TI. Egale à $10^{-2}$ tant que $B_{e}<10^{-3} \mathrm{~T}$, elle devient égale à $B_{e} / B_{s}$ au-delà $\left(B_{s}=0,1 \mathrm{~T}\right)$.

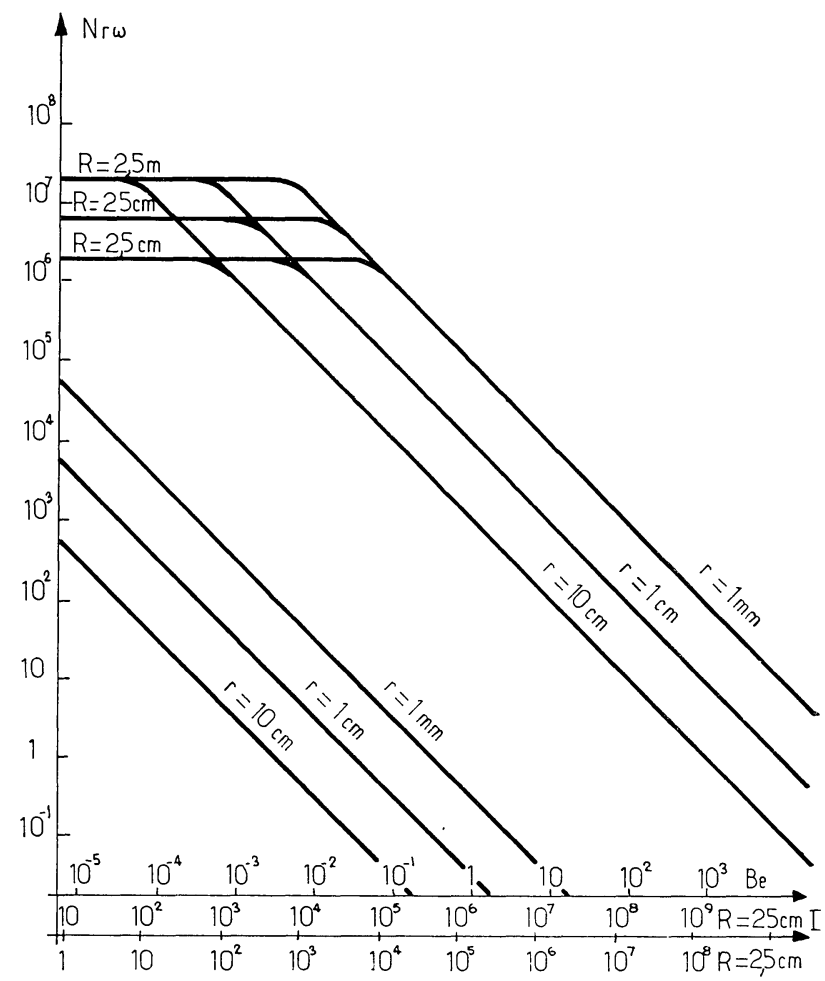

FIG. 4.

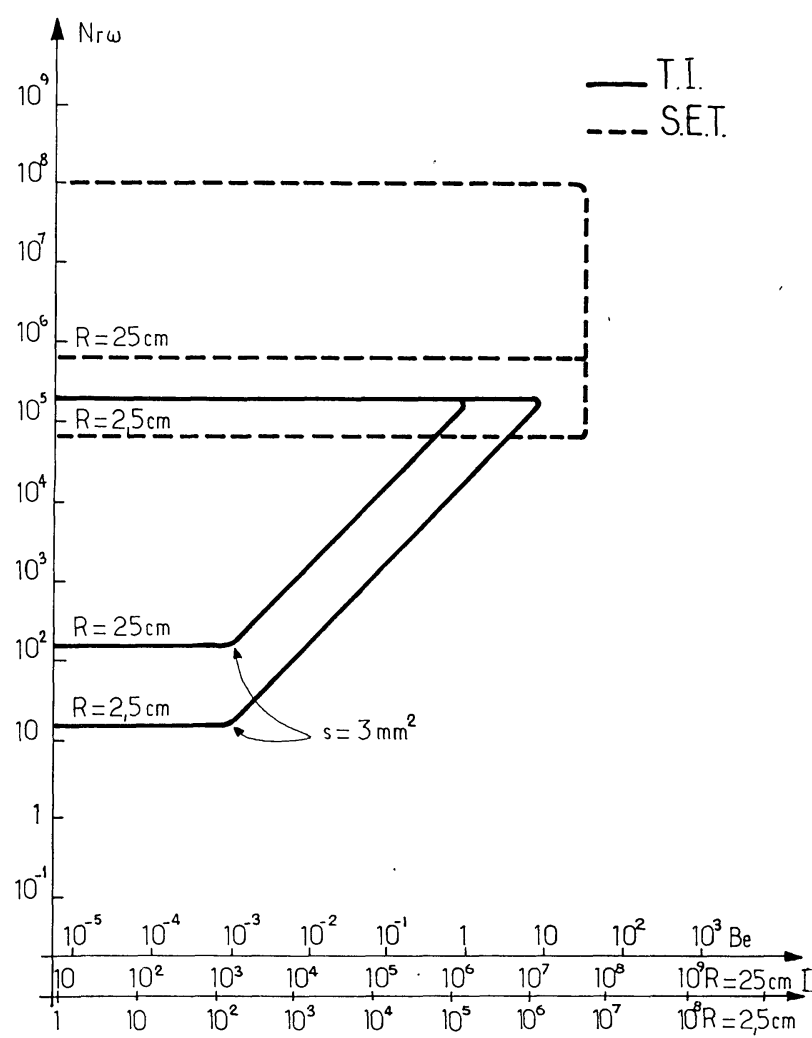

FIG. 5. 
$\omega_{\mathrm{m}}$ est donc donné par :

$$
\frac{\mu_{0} e r \omega_{\mathrm{m}}}{2 \rho}=\frac{\mu_{0}}{2 \rho} \frac{s}{2 \pi R} \cdot N r \omega_{\mathrm{m}}=k .
$$

Le produit $N r \omega_{\mathrm{m}}$ augmente avec $R / s$. Pour que $\omega_{\mathrm{m}}$ soit petit, on a donc intérêt à utiliser un fil de gros diamètre. Nous admettrons pour avoir un ordre de grandeur de la valeur de $N r \omega_{\mathrm{m}}$ que la section maximum du fil facilement utilisable est $s=3 \mathrm{~mm}^{2}$.

Nous avons porté sur le diagramme de la figure 5 la relation (18), la relation (27) pour $s=3 \mathrm{~mm}^{2}$ et diverses valeurs de $R$ ainsi que la condition à un échauffement acceptable $B_{e}<34 \mathrm{~T}$ ((19), RP 1).

Nous voyons immédiatement sur ce diagramme que les bandes passantes des TI et des SET sont étroites, du même ordre de grandeur, centrées sur des fréquences basses pour les TI et hautes pour les SET. Evaluons-les. Nous avons :

$$
\frac{\omega_{\mathrm{M}}}{\omega_{\mathrm{m}}}=\frac{\mu_{0}}{2 \rho} \frac{\sqrt{g} \gamma}{k} \frac{s}{2 \pi R}
$$

soit :

$$
\frac{\omega_{\mathrm{M}}}{\omega_{\mathrm{m}}}=10^{7} \frac{\mathrm{s}}{R} \quad \text { pour une SET }
$$

et :

$\frac{\omega_{\mathrm{M}}}{\omega_{\mathrm{m}}}=2,5 \times 10^{7} \frac{\mathrm{s}}{R} \quad$ pour un TI $\left(B_{e}<10^{-3} \mathrm{~T}\right)$.

Avec la valeur maximum $s=3 \mathrm{~mm}^{2}$ précédemment admise, elles valent donc $30 / R$ pour une SET et $75 / R$ pour un TI ce qui, effectivement, correspond à une bande passante très étroite.

On pourrait augmenter cette bande en prenant un « fil » de section très grande, c'est-à-dire en compliquant de façon appréciable la technologie ; de plus, l'encombrement et le poids des sondes deviendraient dans ce cas très importants surtout en ce qui concerne les TI qui comportent un noyau magnétique.

Ainsi, nous pouvons dire de manière générale que ce type de sonde est mieux adapté à la mesure de l'intensité d'un signal de fréquence donnée qu'à l'étude de la forme d'un signal tout à fait quelconque. En particulier, comme nous le verrons dans le prochain chapitre, les SET qui sont utilisables seulement à fréquence élevée seront toujours très avantageusement remplaçables par des CR ou des SDI. Les TI sont bien adaptés pour les courants d'intensité assez faible de fréquence fixe et peu élevée ; il est d'ailleurs bien connu qu'ils sont couramment utilisés à $50 \mathrm{~Hz}$.

Pour terminer, précisons le domaine d'utilisation des TI. Pour que la bande passante ne soit pas diminuée, il est préférable que $B_{e}$ reste faible $\left(B_{e}<10^{-3}-10^{-2} \mathrm{~T}\right) ;$ le courant à mesurer doit donc être limité à quelques milliers d'ampères.

Si $R=2,5 \mathrm{~cm}, 16<N r \omega<5 \times 10^{4}$.
Prenons par exemple $N=100, r=1 \mathrm{~cm}$, les fréquences $f=\omega / 2 \pi$ mesurables à $1 \%$ ou mieux sont alors telles que :

$$
3 \lesssim f \lesssim 10^{4} .
$$

Si $R=25 \mathrm{~cm}, 160<N r \omega<5 \times 10^{4}$.

Avec $N=100$ et $r=10 \mathrm{~cm}$, on a alors :

$$
3 \lesssim f \lesssim 10^{3} \text {. }
$$

Ces exemples numériques nous montrent clairement que les TI sont bien adaptés à la mesure du courant industriel, mais seulement à ce courant.

3. SONDES A DÉBIT INTERNE (SDI). - Traçons tout d'abord dans les coordonnées $\left(B_{e}, N r \omega\right)$ le diagramme délimitant le domaine d'utilisation des sondes à débit interne (cf. Fig. 6).

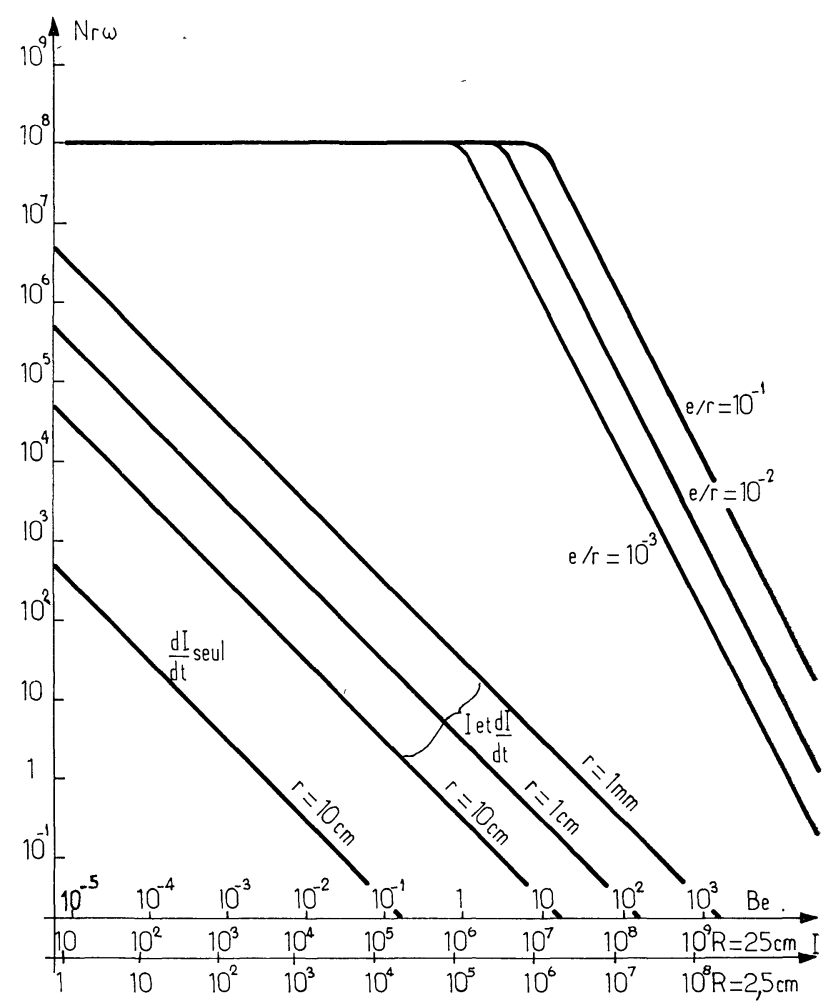

FIG. 6.

En ce qui concerne les hautes fréquences, nous avons montré que les sondes devaient être construites de telle sorte que la relation (20) $\rho R / s=10$ soit vérifiée. Les courbes limitant le domaine d'utilisation sont alors déterminées par les équations :

$$
\begin{aligned}
N r \omega_{\mathrm{M}} & =\sqrt{g} \gamma \quad(\text { relation 18) } \\
r^{2} \omega_{\mathrm{M}} B_{e}^{2} & =\frac{8}{\pi} \rho \bar{\omega} c \Delta T_{0} \quad \text { (relation 30, RP 1). }
\end{aligned}
$$

Cette dernière équation indique que l'échauffement reste inférieur à $\Delta T_{0}=200^{\circ} \mathrm{C}$, ce qui suppose que la résistance du fil varie très peu avec la température. Les alliages nickel-chrome pour lesquels 
$\rho \sim 1,3 \times 10^{-6} \Omega . \mathrm{m}$ ont un coefficient de température très faible et conviennent très bien. De plus, la valeur élevée de leur résistivité permet que $\omega_{M}$ soit grand et, comme nous le verrons, ne présente par ailleurs que des avantages.

Pour traduire la relation ((30), RP 1) dans les coordonnées $B_{e}, N r \omega$, nous remplaçons $\rho$ par son expression tirée de $(20)$

$$
\rho \frac{\mu_{0} \sqrt{g} \gamma}{4 \pi} \cdot \frac{s}{R}=\frac{\mu_{0} \sqrt{g} \gamma}{4 \pi} \cdot \frac{2 \pi e}{N}
$$

ce qui donne :

$$
\left(N r \omega_{\mathrm{M}}\right) \cdot B_{e}^{2}=\frac{4}{\pi} \bar{\omega} c \Delta T_{0} \mu_{0} \sqrt{g} \gamma \frac{e}{r}=1,15 \times 10^{11} \frac{e}{r} .
$$

La limite basse fréquence est donnée par le fait que le signal de mesure doit être plus grand que $U_{\mathrm{m}}((32), \mathrm{RP} 1)$ :

$$
N r_{\mathrm{m}} B_{e}=\frac{U_{\mathrm{m}}}{\pi g r}=\frac{3 \times 10^{-2}}{r} .
$$

Remarquons que cette limite correspond aux mesurés de $J$ et $\mathrm{d} J / \mathrm{d} t$. Dans le cas où l'on ne s'intéresse qu'à $\mathrm{d} J / \mathrm{d} t$ elle est identique à celle obtenue pour les CR, c'est-à-dire $1 / g$ fois plus basse que celle indiquée par (31).

Sur la figure 6 , nous avons porté la relation (18), la relation (30) pour diverses valeurs de $e / r$ et la relation (31) pour diverses valeurs de $r$.

Nous y voyons en particulier que la coupure haute fréquence est déterminée par les phénomènes de propagation dans la sonde en présence d'anisotropie du champ tant que ce dernier n'est pas trop élevé $\left(B_{e} \lesssim 1 \mathrm{~T}\right)$. La bande passante de la SDI se calcule alors facilement. De (18) et (31) nous tirons en effet :

$$
\frac{\omega_{\mathrm{M}}}{\omega_{\mathrm{m}}}=\frac{\pi g^{3 / 2} \gamma}{U_{\mathrm{m}}} r B_{e}=600 \frac{r}{R} I .
$$

La bande passante d'une SDI augmente avec $I$ tant que ce courant ne dépasse pas $10^{5}-10^{6} \mathrm{~A}$. Au-delà, elle décroît lorsque $I$ augmente mais reste grande (de l'ordre de $10^{4}$ pour $B_{e} \simeq 10^{3} \mathrm{~T}$ ). Autrement dit, la bande passante d'une SDI donnée est toujours grande.

Considérons maintenant la façon de construire ces sondes :

- $\omega_{\mathbf{M}}$ étant déterminé par la valeur maximum $\mathrm{du}$ produit $N r \omega_{\mathrm{M}}$, un courant de haute fréquence doit être mesuré avec une sonde de rayon $r$ petit (par exemple $r=1 \mathrm{~mm}$ ) comportant peu de spires (bobinage peu serré).

La valeur maximum de $N r \omega_{M}$ est $10^{8}$; elle est valable lorsque l'intensité du courant n'est pas trop forte et lorsque la sonde vérifie $\rho R / s=10$. Dans ce cas, le fil du bobinage est fin : fil de diamètre $0,2 \mathrm{~mm}$ pour $R=30 \mathrm{~cm}$ et $\rho=10^{6}$. (Remarquons que la condition $\rho R / s=10$ imposerait un fil excessivement fin si le fil était en cuivre et $R$ petit, d'où un intérêt supplémentaire de l'alliage nickel-chrome.)

Calculons la valeur de $e / r$ d'une telle sonde pour savoir quelle courbe donne $\omega_{M}$ aux intensités élevées :

$$
\frac{e}{r}=\frac{N s}{2 \pi R r}=\frac{N}{2 \pi r} \frac{\rho}{10} .
$$

Nous voyons l'intérêt de la valeur élevée de $\rho$ qui augmente la valeur de $e / r$ et conduit à une meilleure réponse haute fréquence lorsque la sonde est utilisée pour des courants plus intenses.

En fait, lorsque la sonde est destinée à mesurer seulement des courants élevés, la valeur de $\omega_{M}$ est donnée par ((30), RP 1). Elle est d'autant plus élevée que $r$ est plus petit et $\rho$ plus grand, mais ne dépend pas des autres paramètres constructifs de la sonde. Il n'est pas indispensable mais préférable qu'elle vérifie $\rho R / s=10$ et $N$ petit, puisque dans ce cas, elle pourra aussi être utilisée dans les meilleures conditions. pour des courants plus faibles.

En résumé, les SDI construites pour les hautes fréquences ont une grande bande passante, un poids et un encombrement très faibles.

En ce qui concerne la mesure des courants basse fréquence, il est préférable de choisir une SDI pour laquelle $N$ et $r$ sont grands, car $N r \omega_{\mathrm{m}}$ est alors petit et $\omega_{\mathrm{m}}$ aussi.

IV. Comparaison des divers types de sonde. Conclusion. - Nous venons de déterminer les domaines

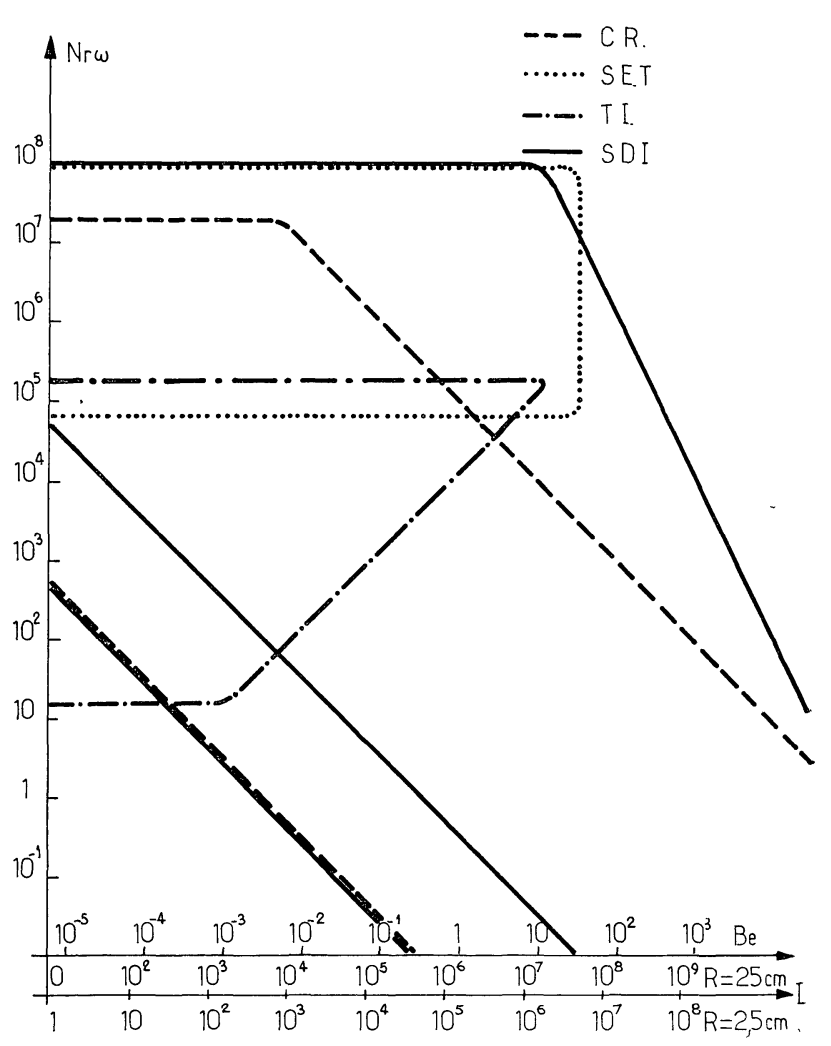

FIG. 7. 
d'utilisation des divers types de sondes et nous nous proposons de les comparer. Pour cela, nous avons porté sur un même diagramme (Fig. 7) les courbes qui déterminent les possibilités extrêmes d'utilisation de chaque type de sonde (cf. Fig. 4, 5 et 6). Notons d'ailleurs que ces courbes, correspondent en général à des structures différentes pour les mesures HF et $\mathrm{BF}$.

Nous voyons alors, en ce qui concerne les hautes fréquences, que les SDI sont en pratique supérieures aux deux autres types de sondes utilisables en HF : les CR et les SET. En particulier, bien que les SET soient meilleures dans un très petit domaine, elles sont à exclure étant donné leur très faible bande passante et leurs poids et encombrement beaucoup plus grands que celui des SDI et CR. Les CR ont une bonne bande passante mais ne mesurent que $\mathrm{d} J / \mathrm{d} t$ et leur limite supérieure en fréquence est nettement plus basse que pour les SDI.

En ce qui concerne les basses fréquences, les sondes utilisables sont les CR, les SDI et les TI. Les CR et les SDI sont équivalentes pour la mesure de $\mathrm{d} J / \mathrm{d} t$; les SDI mesurent en plus $J$ mais dans ce cas leur coupure basse fréquence est $1 / g$ fois plus haute. Comparons donc la limite $\mathrm{BF}$ de mesure de $\mathrm{d} J / \mathrm{d} t$ par une $\mathrm{CR}$ ou une SDI à la limite $\mathrm{BF}$ de mesure de $J$ par un TI. Sur la figure 7, nous distinguons immédiatement deux cas :

- Si le courant est élevé, les SDI et CR répondent plus bas en fréquence.

- S'il est faible, les TI répondent mieux.

En pratique, comme nous l'avons déjà dit, les TI sont bien adaptés à la mesure de courants de fréquence industrielle lorsque l'intensité ne dépasse pas quelques milliers d'ampères et ce sont alors les meilleures sondes de mesure inductive. Pour les courants de plus forte intensité, les SDI sont préférables. Pour des courants de basse fréquence et de faible intensité, les sondes inductives que nous avons étudiées ne conviennent pas.

En conclusion, lorsqu'on recherche les structures des sondes inductives les mieux adaptées à la mesure des courants variables, deux types de sondes s'imposent :

- les transformateurs d'intensité pour la mesure des courants industriels (faibles à variations lentes);

- les sondes à débit interne pour tous les autres types de courants variables.

Remerciements. - Nous tenons à remercier M. Guy Delouya pour sa lecture critique du manuscrit et pour les suggestions qu'il nous a faites.

\section{Bibliographie}

RP 1 - [1] Rioux-Damidau (F.) et Rioux (C.), " Etude générale des sondes de mesure inductive de courants. I. Description des principaux types de sondes. »

Rev. Phys. Appl. 1972, 7, 303. 